\title{
Unusual Invasıon of Trichilemmal Umors: Two Case Reports
}

\author{
Mehtap Karamese, Ahmet Akatekin, Malik Abaci, Zekeriya Tosun, Mustafa Keskin
}

Reconstructive and Aesthetic Surgery, Department of Plastic, Selcuklu Faculty of Medicine, Selcuk University, Konya, Turkey. Email: mehtapef@yahoo.com, \{ahmetakatekin, maablaicki\}@gmail.com, \{ztosun,drmkeskin\}@hotmail.com

Received April $4^{\text {th }}$, 2012; revised May $7^{\text {th }}, 2012$; accepted June $6^{\text {th }}, 2012$

\begin{abstract}
Background: Proliferating trichilemmal tumors are slow-growing lobulated masses most commonly found on the scalp of elderly women. Due to the locally invasive nature of the lesion, the treatment is complete excision of the tumor with tumor-free margins. Methods: We present two cases of trichilemmal tumors that exhibited aggressive local invasion across tissue planes. The first case had dural invasion, which needed dural reconstruction. The second case had muscle invasion, which required wide resection. Results: Sixteen months after their surgeries, the patients are in good health without any recurrence of tumors. Conclusion: Trichilemmal tumors may exhibit aggressive local invasion across tissue planes and even penetrate intracranially, causing considerable morbidity and mortality. The possibility of dural and muscle invasion must be kept in mind in order to achieve successful treatment results. A close clinical follow-up is judicious for detecting recurrences or metastases.
\end{abstract}

Keywords: Trichilemmal Tumor; Dural Invasion

\section{Introduction}

Proliferative trichilemmal tumors are rare neoplasms that develop from the external root sheath of a hair follicle. These tumors arise mainly in areas of dense hair follicle concentration: 90\% occur on the scalp [1]. The usual clinical presentation is a subcutaneous cystic nodule. Patients most often present with a solitary lesion, but multiple lesions are encountered at times. A trichilemmal tumor has a benign clinical course, but exhibits a malignant and invasive histological appearance [2]. Dural invasions or muscle invasions of trichilemmal tumors have been very rarely reported. However, here were port a dural invasion and a muscle invasion in two cases of trichilemmal tumors.

\section{Case Report No. 1}

A 49-year-old man presented with a mass in his scalp. He had burned his scalp with hot water 25 years ago. He first noticed this mass four years ago. The lesion had been growing rapidly for the previous six months. Upon examination, the lesion was $8 \times 6 \times 5 \mathrm{~cm}$ in size with irregular margins and superficial erosion (Figure 1). No other regional lymphadenopathy was detected. The brain computerized tomography revealed a mass with dural invasion (Figure 2). A wide excision was performed under general anesthesia. The dura was excised and re- placed with a dural graft by a neurosurgeon. A bone defect was replaced with a split calvarial graft. Scalp reconstruction was done with a bipedicled scalp flap. Histopathological diagnosis of the mass determined it was a malign proliferative trichilemmal tumor. The local oncology committee did not recommend chemotherapy. At the 16-month follow-up, there was no evidence of local recurrence or metastasis (Figure 3) [3].

\section{Case Report No. 2}

A 47-year-old man presented with a huge mass on his upper back. He first noticed this mass 10 years ago. The lesion had been growing rapidly for the previous five months. Upon examination, the $16 \times 17 \mathrm{~cm}$ pain less non-tender mass was palpated on the trapezius muscle (Figure 4). The lesion had occurred spontaneously and was not associated with any history of trauma, and there was no past history of a similar tumor any where on his body. The tumor was not fixed to the underlying bones. Magnetic resonance imaging showed the tumor had infiltrated the underlying muscle with undistinguishable borders (Figure 5). A wide excision of the tumor was performed under general anesthesia. Muscle fibers that had been infiltrated by tumor were excised widely. Reconstruction of the defect required a skin graft. The histopathological analysis determined that the tumor was a proliferative trichilemmal tumor. Allcut magrins were 


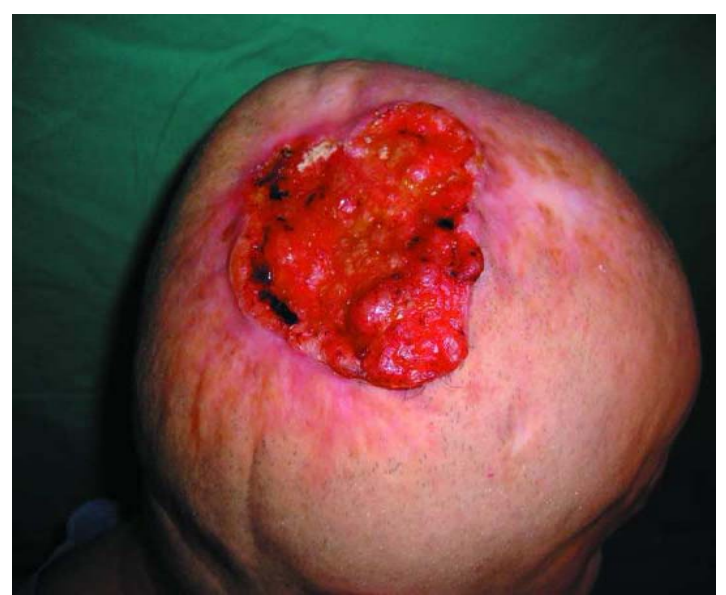

Figure 1. Preoperative photograph of the tumor in case 1.

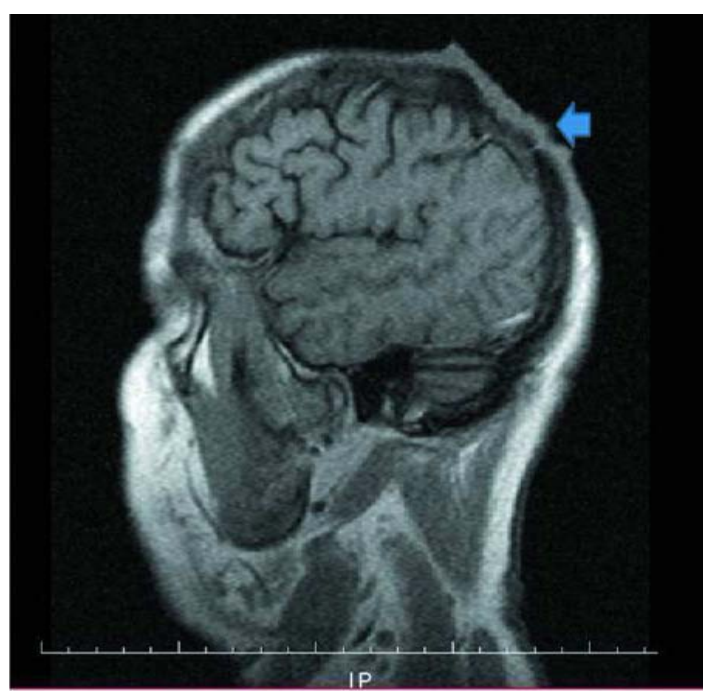

Figure 2. The brain computerized tomography revealing a mass with dural invasion.

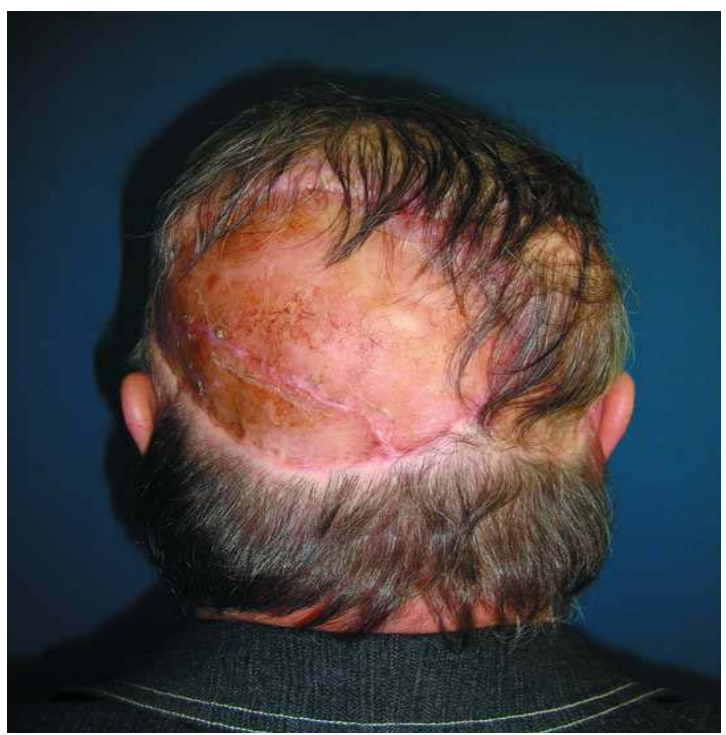

Figure 3. Postoperative photograph of the patient.

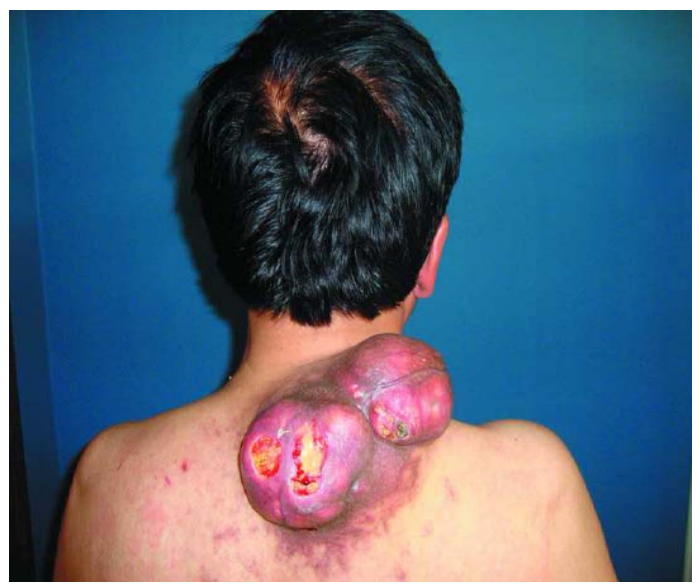

Figure 4. Preoperative photograph of the mass in case 2.

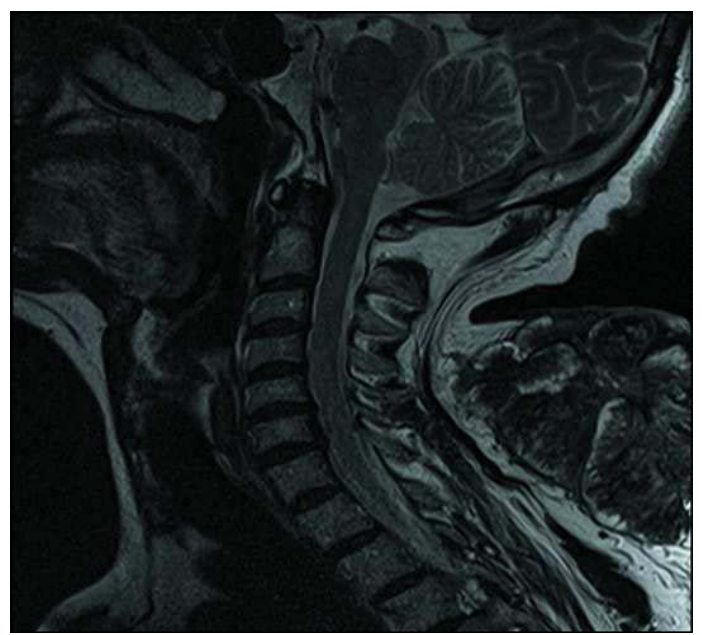

Figure 5. Magnetic resonance imaging showing the tumor had infiltrated the underlying muscle with undistinguishable borders.

free and the base was one mm away from the tumor. At to 10 -month follow-up, there was no local recurrence (Figure 6).

\section{Discussion}

A trichilemmal tumor is an unusual neoplasm. It originates from the hair follicle cells, And the differential diagnosis include sother skin carcinomas. The common sites include the scalp, forehead, and neck, which are sun-exposed are as. Women over 40 years of age are the most commonly affected patients. Grossly, the tumors have been described as exophytic, ulcerated, polypoid, or nodular lesions that may be keratotic. A trichilemmal tumor usually has an indolent clinical course. Proliferating trichilemmal tumors (PTT) usually arise in a preexisting trichilemmal cyst [3]. More aggressive biological behavior is seen in the malignant proliferating trichilemmal tumors (MPTT); these lesions present significant morbidity and potential mortality. Surgery is considered the 


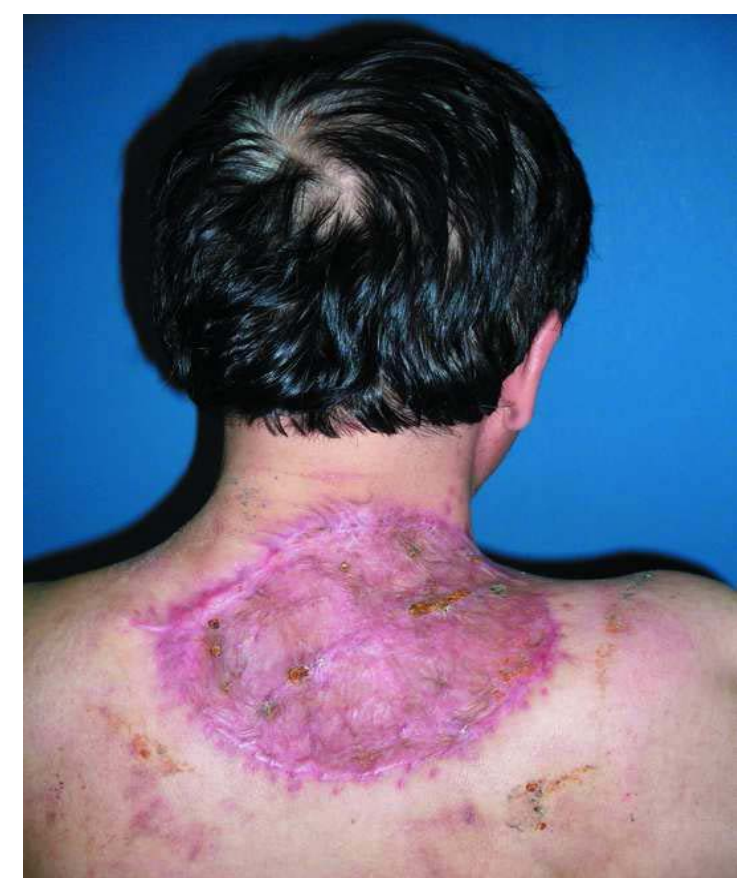

Figure 6. Photograph of the patient in 10-month follow-up.

treatment of choice for trichilemmal tumorsand periodic surveillance without adjuvant therapy is generally sufficient. The differential diagnosis of PTT includes basal cell carcinoma, sebaceous carcinoma, clear cell hydradenocarcinoma, and cutaneous metastasis of renal cell carcinomas [4]. A trichilemmal tumor may exhibit aggressive local invasion across tissue planes and even intracranially, causing considerable morbidity and mortality. Complete resection of the tumor at the first instance, followed by precisere construction of there maining defect, may give the patient the best chance of being cured. Close clinical follow-up is judicious for detecting recurrences or metastases. The cases in this report are both men. They both had an invasive tumor. Although There are some reports of metastatic cases and death from these tumors in the literature, the invasion of the dura is very rare $[1,5,6]$. The first case had dural invasion, which required dural reconstruction. Second case had muscle invasion. Muscle invasion is also rare [2,4]. It is known that MPTT are apt to recurrant to metastasize. Patients should have follow-up examinations for recurrences. Treatment of the PTT is the goal of the plastic surgery. Excision, dermabrasion, electrodessication, $\mathrm{CO}_{2}$ (carbondioxide) laser, cryotherapy, radiotherapy, ortopical applications of aspirin derivatives are reported as the treatment modalities for trichilemmal tumors. For the unwelcome recurrence rates and the risk of malignant transformation of these tumors, a wide local excision is the favored method of treatment [7]. As part of the management of these lesions, reconstruction of large scalp and/or skull defects often poses difficulties and significant sur- gical challenges. Total scalp excision with reconstruction is thetreatment of choice. If the histological diagnosis reveals a malign PTT, more aggressive therapeutic measures such as nodal dissection, radiotherapy or chemotherapy should be considered in addition to wide local excision. The cisplatin, adriamycin, and vindesine (CAV) regimen, previously used for treatment of highly advanced cases of squamous cell carcinoma (SCC), has been used to treat MPTT with very limited success $[5,8]$. Owing to the potentially aggressive behavior of MPTT and the possibility of late recurrence, frequent clinical follow-up and lymph node examinations are advised.

\section{Conclusion}

Consequently, upon presentation of an indurate noduleor an ulcerated mass on the scalp, it is necessary to consider the possibility of a cancerous lesion. It is important to perform a total excision of the tumor mass, with tumorfree margins, and to offer careful follow-ups to all patients. Although trichilemmal carcinoma is a malignant tumor that usually does not metastasize, despite local regional recurrence, the possibility of dural and muscle invasion must be kept in mind in order to achieve successful treatment results [6].

\section{REFERENCES}

[1] H. S. Yi, S. J. Sym, J. Park, et al., "Recurrent and Metastatic Trichilemmal Carcinoma of the Skin over the Thigh: A Case Report,” Cancer Research and Treatment, Vol. 42, No. 3, 2010, pp. 176-179. doi:10.4143/crt.2010.42.3.176

[2] A. K. Satyaprakash, D. J. Sheehan and O. P. Sangüeza, "Proliferating Trichilemmal Tumors; A Review of the Literature,” Dermatologic Surgery, Vol. 33, No. 9, 2007, pp. 1102-1108. doi:10.1111/j.1524-4725.2007.33225.X

[3] J. H. Lee, Y. W. Shin, Y. H. Oh, et al., "Trichilemmal Carcinoma of the Upper Eyelid: A Case Report,” Korean Journal of Ophthalmology, Vol. 23, No. 4, 2009, pp. 301305. doi:10.3341/kjo.2009.23.4.301

[4] J. Ye, O. Nappi, P. E. Swanson, et al., "Proliferating Pilar Tumors. A Clinicopathologic Study of 76 Cases with a Proposal for Definition of Benign and Malignant Variants," American Journal of Clinical Pathology, Vol. 122, 2004, pp. 566-574. doi:10.1309/0XLEGFQ64XYJU4G6

[5] O. Makiese, et al., "Huge Proliferating Trichilemmal Tumors of the Scalp: Report of Sixcases," Plastic and Reconstructive Surgery, Vol. 126, No. 1, 2010, pp. 18e-9e. doi:10.1097/PRS.0b013e3181dbc48e

[6] S. B. Bae, et al., "A Case of Malignant Proliferating Trichilemmoma of the Scalp with Multiple Metastases," Korean Journal of Internal Medicine, Vol. 16, No. 1, 2001, pp. 40-43.

[7] Y. Komuro, T. Takedai and K. Tagawa, "Proliferating Trichilemmal Tumor on the Dorsum of the Hand," Annals of Plastic Surgery, Vol. 34, No. 6, 1995, pp. 657-659. 
doi:10.1097/00000637-199506000-00017

[8] M. Siddha, et al., "Malignant Pilar Tumor of the Scalp: A Case Report and Review of Literature,” Journal of Can- cer Research and Therapeutics, Vol. 3, No. 4, 2007, pp. 240-243. doi:10.4103/0973-1482.39001 